\title{
Colloidal Flower-Shaped Iron Oxide Nanoparticles: Synthesis Strategies and Coatings
}

Gavilán, Helena ; Kowalski, Anja ; Heinke, David ; Sugunan, Abhilash ; Sommertune, Jens ; Varón, Miriam; Bogart, Lara K. ; Posth, Oliver ; Zeng, Lunjie ; González-Alonso, David

Total number of authors:

21

Published in:

Particle \& Particle Systems Characterization

Link to article, DOI:

10.1002/ppsc.201700094

Publication date:

2017

Document Version

Peer reviewed version

Link back to DTU Orbit

Citation (APA):

Gavilán, H., Kowalski, A., Heinke, D., Sugunan, A., Sommertune, J., Varón, M., Bogart, L. K., Posth, O., Zeng, L., González-Alonso, D., Balceris, C., Fock, J., Wetterskog, E., Frandsen, C., Gehrke, N., Grüttner, C., Fornara, A., Ludwig, F., Veintemillas-Verdaguer, S., ... Morales, M. P. (2017). Colloidal Flower-Shaped Iron Oxide Nanoparticles: Synthesis Strategies and Coatings. Particle \& Particle Systems Characterization, 34(7). https://doi.org/10.1002/ppsc.201700094

\section{General rights}

Copyright and moral rights for the publications made accessible in the public portal are retained by the authors and/or other copyright owners and it is a condition of accessing publications that users recognise and abide by the legal requirements associated with these rights.

- Users may download and print one copy of any publication from the public portal for the purpose of private study or research.

- You may not further distribute the material or use it for any profit-making activity or commercial gain

- You may freely distribute the URL identifying the publication in the public portal 


\section{Particle \\ \& Particle Systems Characterization}

Postfach 101161 69451 Weinheim

Germany

Courier services:

Boschstraße 12

69469 Weinheim

Germany

Tel.: (+49) 6201606235 / 432

Fax: (+49) 6201606500

E-mail: particle@wiley.com
Dear Author,

Please correct your galley proofs carefully and return them no more than three days after the page proofs have been received.

If you have not used the PXE system before, please view the Tutorial before checking your proofs: http://wileypxe.aptaracorp.com/pxewileyvch/ UserDocument/UserGuide/WileyPXE5 Author Instructions.pdf

Please note any queries that require your attention. These are indicated with red Qs in the pdf or highlighted as yellow queries in the "Edit" window.

Please pay particular close attention to the following, as no further corrections can be made once the article is published online:

- Names of all authors present and spelled correctly

- Titles of authors are correct (Prof. or Dr. only: please note, Prof. Dr. is not used in the journals)

- Addresses of all authors and e-mail address of the corresponding author are correct and up-to-date

- Funding bodies have been included and grant numbers are accurate
- The Title of the article is OK

- All figures are correctly included

- Equations are typeset correctly

Note that figure resolution in the PXE system is deliberately lower to reduce loading times. This will be optimized before the article is published online.

Please send any additional information, such as figures or other display items, to particle@wiley.com, and please also indicate this clearly in the PXE "Edit" window by inserting a comment using the query tool.

Reprints may be ordered by filling out the accompanying form.

Return the reprint order form by e-mail with the corrected proofs, to Wiley- $\mathrm{VCH}$ : particle@wiley.com

Please limit corrections to errors already in the text. Costs incurred for any further changes will be charged to the author, unless such changes have been agreed upon by the editor.

The editors reserve the right to publish your article without your corrections if the proofs do not arrive in time. Note that the author is liable for damages arising from incorrect statements, including misprints. 


\section{Particle \\ Particle Systems Characterization}

Reprint Order Form 2017
Editorial Office:

Wiley-VCH Verlag

Boschstrasse 12

69469 Weinheim, Germany

Tel.: +49 (0) 6201606235 or 432

Fax: +49 (0) 6201606500

Email: particle@wiley.com

\section{Short DOI: ppsc.}

Please send me and bill me for

no. of Reprints via $\quad \square$ airmail (+ 25 Euro)

Please send me and bill me for a

$\square$ high-resolution PDF file (330 Euro).

My Email address:

Please note: It is not permitted to present the PDF file on the internet or on company homepages.

\section{Information regarding VAT}

Please note that from German sales tax point of view, the charge for Reprints, Issues or Posters is considered as "supply of goods" and therefore, in general, such delivery is a subject to German sales tax. However, this regulation has no impact on customers located outside of the European Union. Deliveries to customers outside the Community are automatically tax-exempt. Deliveries within the Community to institutional customers outside of Germany are exempted from the German tax (VAT) only if the customer provides the supplier with his/her VAT number. The VAT number (value added tax identification number) is a tax registration number used in the countries of the European Union to identify corporate entities doing business there. It starts with a country code (e.g. FR for France, GB for Great Britain) and follows by numbers.

VAT no.:

(Institutes / companies in EU countries only)
Delivery address / Invoice address:

Name of recipient, University, Institute, Street name and Street number, Postal Code, Country

\section{Date and Signature:}

Credit Card Payment (optional) -You will receive an invoice.

VISA, MasterCard, AMERICAN EXPRESS

Please use the Credit Card Token Generator located at the website below to create a token for secure payment. The token will be used instead of your credit card number.

Credit Card Token Generator:

https://www.wiley-vch.de/editorial production/index.php

Please transfer your token number to the space below.

\section{Credit Card Token Number}

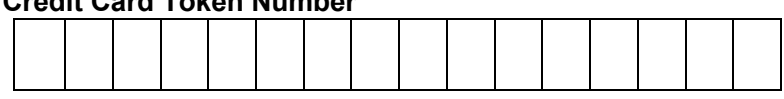

Purchase Order No.:

Price list for reprints (The prices include mailing and handling charges. All Wiley-VCH prices are exclusive of VAT)

\begin{tabular}{c|cccccc}
\hline No. of pages & Price (in Euro) for orders of \\
& 50 copies & 100 copies & 150 copies & 200 copies & 300 copies & 500 copies \\
\hline $1-4$ & 345 & 395 & 425 & 445 & 548 & 752 \\
$5-8$ & 490 & 573 & 608 & 636 & 784 & 1077 \\
$9-12$ & 640 & 739 & 786 & 824 & 1016 & 1396 \\
$13-16$ & 780 & 900 & 958 & 1004 & 1237 & 1701 \\
$17-20$ & 930 & 1070 & 1138 & 1196 & 1489 & 2022 \\
\hline for every additional 4 & 147 & 169 & 175 & 188 & 231 & 315 \\
\hline
\end{tabular}

Wiley-VCH Verlag GmbH \& Co. KGaA; Location of the Company: Weinheim, Germany;

Trade Register: Mannheim, HRB 432833, Chairman of the Board: Mark Allin

General Partner: John Wiley \& Sons GmbH, Location: Weinheim, Germany

Trade Register Mannheim, HRB 432296, Managing Director: Sabine Steinbach 


\section{FULL PAPER}

$\mathbf{x x x x}$

H. Gavilán,* A. Kowalski, D. Heinke, A. Sugunan, J. Sommertune, M. Varón, L. K. Bogart, O. Posth, L. Zeng, D.

González-Alonso, C. Balceris, J. Fock, E. Wetterskog, C. Frandsen, N. Gehrke, C. Grüttner, A. Fornara, F. Ludwig, S.

Veintemillas-Verdaguer, C. Johansson, M. P. Morales* $x-x x$

Colloidal Flower-Shaped Iron Oxide Nanoparticles: Synthesis Strategies and Coatings

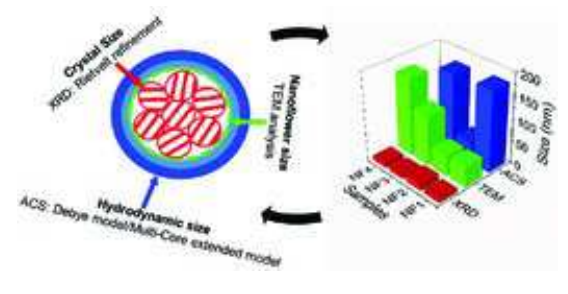

Different strategies to obtain multi-core nanoparticles with flower-shaped structure in the size range of $25-100 \mathrm{~nm}$ are examined. The core size within the nanoflower is measured, calculated and modeled through structural and magnetic characterization, revealing multicores nanoparticles that have very different intra- and interparticle interactions. We identified the relevant synthesis parameters determining the core assembly. 


\title{
Colloidal Flower-Shaped Iron Oxide Nanoparticles: Synthesis Strategies and Coatings
}

\author{
Helena Gavilán,* Anja Kowalski, David Heinke, Abhilash Sugunan, Jens Sommertune, \\ Miriam Varón, Lara K. Bogart, Oliver Posth, Lunjie Zeng, David González-Alonso, \\ Christoph Balceris, Jeppe Fock, Erik Wetterskog, Cathrine Frandsen, Nicole Gehrke, \\ Cordula Grüttner, Andrea Fornara, Frank Ludwig, Sabino Veintemillas-Verdaguer, \\ Christer Johansson, and M. Puerto Morales*
}

The assembly of magnetic cores into regular structures may notably influence the properties displayed by a magnetic colloid. In this work, key synthesis parameters driving the self-assembly process capable of organizing colloidal magnetic cores into highly regular and reproducible multi-core nanoparticles are determined. In addition, a self-consistent picture that explains the collective magnetic properties exhibited by these complex assemblies is achieved through structural, colloidal, and magnetic means. For this purpose, different strategies to obtain flower-shaped iron oxide assemblies in the size range 25-100 nm are examined. The routes are based on the partial oxidation of $\mathrm{Fe}(\mathrm{OH})_{2}$, polyol-mediated synthesis or the reduction of iron acetylacetonate. The nanoparticles are functionalized either with dextran, citric acid, or alternatively embedded in polystyrene and their long-term stability is assessed. The core size is measured, calculated, and modeled using both structural and magnetic means while the Debye model and multi-core extended model are used to study interparticle interactions. This is the first step toward standardized protocols of synthesis and characterization of flower-shaped nanoparticles.

\section{H. Gavilán, Dr. M. P. Morales}

Department of Energy, Environment and Health Instituto de Ciencia de Materiales de Madrid, ICMM/CSIC

Sor Juana Inés de la Cruz 3, 28049, Madrid, Spain

A. Kowalski, Dr. C. Grüttner

Micromod Partikeltechnologie $\mathrm{GmbH}$

Friedrich-Barnewitz-Str. 4, D-18119, Rostock, Germany

D. Heinke, Dr. N. Gehrke

NanoPET Pharma GmbH

, D-10115, Berlin, Germany

Dr. A. Sugunan, Dr. J. Sommertune, Prof. A. Fornara

SP Technical Research Institute of Sweden

Box 5607, SE-114 86, Stockholm, Sweden

Dr. M. Varón, Prof. C. Frandsen

Department of Physics

DTU

Fysikvej, 2800, Kongens Lyngby, Denmark

Dr. L. K. Bogart

UCL Healthcare Biomagnetics Laboratory

$\mathrm{UCL}$

21 Albemarle Street London, W1S 4BS, UK

\section{Dr. O. Posth}

Physikalisch-Technische Bundesanstalt

Abbestr. 2-12, 10587, Berlin, Germany

Dr. L. Zeng

Department of Applied Physics

Chalmers University of Technology

Gothenburg, SE-41296, Sweden

Dr. D. González-Alonso

Department of CITIMAC

Faculty of Science

University of Cantabria

, 39005, Santander, Spain

C. Balceris, Dr. F. Ludwig

Institute of Electrical Measurement and Fundamental Electrical

Engineering

TU Braunschweig, D-38106, Germany

Dr. J. Fock

Department of Micro- and Nanotechnology

DTU

, DK-2800, Kongens Lyngby, Denmark

Dr. E. Wetterskog

Department of Engineering Sciences

Ångström Laboratory 


\section{Introduction}

Despite the progress in colloidal self-assembly of organic ${ }^{[1]}$ or inorganic ${ }^{[2,3]}$ building blocks to form close-packed structures such as colloidal crystals, ${ }^{[4]}$ there are only a few reports of controlled assembly of ordered nanoparticles in suspension. ${ }^{[5]}$ In this matter, many fundamental aspects remain unknown and often, there is no predictive description of the ensemble behaviour or the origin of the forces driving aggregation. ${ }^{[6,7]}$ Nonetheless, aggregation is a general mechanism of particle formation now recognized as a common growth phenomenon for many different monodisperse nanoparticles, ${ }^{\left[{ }^{[-12]}\right.}$ in contrast to the monomer by monomer addition that is described in classical models.

In absence of templates, interfaces or external fields, the selfassembly process in solution is governed by the balance of attractive and repulsive forces. Magnetic nano-particles (MNPs) are a priori - different due to additional forces that arise as a consequence of their magnetic moment. These interactions can be either intraparticle ex-change interactions, or long-range magnetostatic dipolar forces between particles. Exchange interactions between cores of a multi-core particle may lead to the so-called "superferrimagnetic" behaviour, ${ }^{[13]}$ exhibiting large magnetic moment and weak remanence in zero field, and thus, having low tendency to form agglomerates. On the other hand, dipolar interactions between particles with sufficient high moment account for the formation of configurations such as chains, which may change strongly the magnetic properties of the colloid. ${ }^{[14]}$ As a consequence, the assembly of MNPs in multi-core structures can, in some cases, give rise to collective magnetic properties, which yields microscopic magnetic behaviour that is very different from single-core nanoparticles or bulk materials. ${ }^{[15,16]}$ Such multi-core nanoparticles are currently of great interest in many different areas such as catalysis, ${ }^{[17]}$ ferrofluids and rheology, ${ }^{[18]}$ as well as bioapplications, which are mainly focused on iron oxide nanoparticles (magnetite or maghemite) because of their low toxicity. ${ }^{[19]}$ Colloids made of magnetic multi-core nanoparticles show high NMR relaxivity (r2), ${ }^{[20]}$ high MPI signals, ${ }^{[21]}$ high specific absorption rate of AC field (SAR), ${ }^{[22-24]}$ high magnetic moment when manipulated with an external magnet ${ }^{[25]}$ and enhanced performance as theranostic agents. ${ }^{[26-28]}$

Herein, we analyse the key synthesis parameters driving the self-assembly process capable of organizing colloidal magnetic cores into highly regular and reproducible multi-core nanoparticles showing the so called "superferrimagnetic state" due to exchange interactions. For that purpose we have conducted a comparison of four different synthesis of colloidal magnetic multi-

Uppsala University

, Sweden

Dr. S. Veintemillas-Verdaguer

Instituto de Ciencia de Materiales de Madrid ICMM/CSIC

Sor Juana Inés de la Cruz 3, 28049, Madrid, Spain

Prof. C. Johansson

Acreo Swedish ICT AB

P.O. Box 53071, SE-400 14, Göteborg, Sweden

Correspondence to: H. Gavilán (E-mail: helena_gr@icmm.csic.es), Dr. M. P. Morales (E-mail: puerto@icmm.csic.es)

DOI: 10.1002/ppsc.201700094

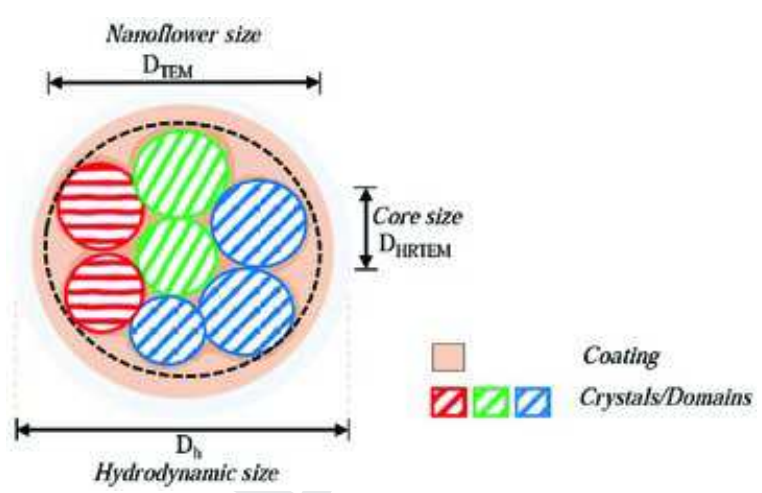

Scheme 1. Flower-shaped multi-core nanoparticle: Nanoflower and core size have been determined through TEM and HRTEM, respectively, and has a final hydrodynamic size in solution. The term crystal/domain is designated as cores that share crystal alignment.

core structures called flower-shaped nanoparticles (Scheme 1), consisting of iron oxide cores that are aggregated to form isometric $3 \mathrm{D}$ arrangements. Out of the numerous synthesis strategies re-ported in the literature, ${ }^{[5]}$ we have focused our investigation on those that either produce multi-core particles in the singledomain region, i.e. smaller than $50 \mathrm{~nm}$, or above the multidomain limit, i.e. larger than $100 \mathrm{~nm}$. In this work, some used approaches describe the in situ formation of MNPs and assembly in the presence of molecules or polymers, while others describe first the assembly of the formed MNPs and its further polymer coating, encapsulation or embedment. By comparing synthesis approaches we have been able to gain a better understanding into the nature of this self-assembly process that lead to multicore magnetic nanoparticles with controllable size, shape and collective behaviour. Moreover, we have been able to determine to which extent the systems can be tuned by choice of synthesis conditions yielding core aggregation and colloidal stability.

Comprehensive structural and magnetic characterisation of colloidal dispersions and freeze-dried powders has been performed following standardized protocols to facilitate comparison of these structures. ${ }^{[29]}$ This is the first step toward standardization of synthesis and characterization of these nanoparticles, which is an important concern and demand nowadays. ${ }^{[30]}$ First, we analyse the core arrangement within the particle and secondly we analyse interparticle interactions. A key parameter to understand the behaviour of the colloid is the degree of fusion of the cores within the nanoflowers, specifically whether they are in direct contact and if so, if they share crystalline alignment. ${ }^{[31]}$ Secondly, we analyse the interparticle interactions, ${ }^{[32]}$ which are minimized by steric and/or electrostatic repulsion due to the surface coatings (dextran, citric acid) or alternatively by embedding the cores on surfactant stabilized polystyrene beads. Surface modification of the flower-shaped nanoparticles provides colloidal stability in water and specific functionalization, which is of utmost importance for their successful application in the nano-bio area. ${ }^{[33]}$ 


\begin{tabular}{|c|c|c|c|c|}
\hline & Strategy 1 & Strategy 2 & Strategy 3 & Strategy 4 \\
\hline Precursor & $\mathrm{Fe}$ (II) & $\mathrm{Fe}$ (III)/Fe (III) & \multicolumn{2}{|c|}{$\mathrm{Fe}$ (III) } \\
\hline $\begin{array}{r}\text { Reducing or } \\
\text { Oxidizing agent }\end{array}$ & $\begin{array}{c}\text { Potassium nitrate } \\
\mathrm{KNO}_{3}\end{array}$ & $\begin{array}{l}\text { Polvol } \\
\mathrm{C}_{2} \mathrm{H}_{20}+2 \mathrm{O}_{n}\end{array}$ & \multirow{3}{*}{$\begin{array}{c}\begin{array}{c}\text { Polyol } \\
\mathrm{C}_{n} \mathrm{H}_{2 n}+2 \mathrm{O}_{n}\end{array} \\
\text { EG } \\
\text { Citric acid }\end{array}$} & $\begin{array}{l}\text { Sodium borohydride } \\
\mathrm{NaBH}_{4}\end{array}$ \\
\hline Media & Water & DEG/NMDEA & & THF/Water \\
\hline \multirow{3}{*}{$\begin{array}{r}\text { In-situ coating } \\
\text { Extra-step } \\
\text { for coating }\end{array}$} & Dextran & $\stackrel{\text { None }}{1}$ & & \multirow{2}{*}{$\frac{1}{\text { ESE Process }}$} \\
\hline & & HPH Process. & & \\
\hline & & Dextran & & sityrene \\
\hline \multirow{3}{*}{$\begin{array}{l}\text { Flower-shaped } \\
\text { Nanoparticles }\end{array}$} & ${ }^{8}$ & (8) & & \\
\hline & $N E=1$ & & & $N \in-A$ \\
\hline & $N F=1$ & & & \\
\hline
\end{tabular}

Scheme 2. Strategies followed to obtain flower-shaped nanoparticles. $\mathrm{N}$-methyl diethanolamine, $\mathrm{HPH}$ process stands for high-pressure homogenization coating and ESE process stands for emulsion solvent evaporation.

\section{Results and Discussion}

\subsection{Key Parameters Controlling Self-Assembly}

The Strategy 1 (Scheme 2) involves the oxidative aging of an $\mathrm{Fe}(\mathrm{OH})_{2}$ intermediate in water in the presence of dextran $(90$ ${ }^{\circ} \mathrm{C} / 4 \mathrm{~h}$ ), to yield NF1. Table 1 includes the mean size of the particle and core determined through TEM and HRTEM, respectively. Nanoflowers produced in this way have a mean diameter of $46 \mathrm{~nm}$ and they are composed of $7 \mathrm{~nm}$ cores that are loosely packed together (Figure 1). HRTEM images suggest that the cores share the same crystallographic orientation in some areas of the particle but not throughout the whole particle. It has previously been reported that for this reaction the excess of $\mathrm{OH}^{-}$or $\mathrm{Fe}^{2+}$ in the media defines the mechanism of particle growth and therefore the formation of single or multi-core particles. ${ }^{[34,35]}$ In our approach, an excess of $\mathrm{OH}^{-}$was used to move the $\mathrm{pH}$ away from the magnetite isoelectric point $(\sim 6.5),{ }^{[36]}$ thus charging the surface of the initial growing nuclei keeping them apart and resulting in single-core particles. ${ }^{[35,37,38]}$ The use of in-situ dextran coating, however, causes a reduction in surface charge density of the growing nuclei promoting its approach and aggregation. We observe that flower-shaped nanoparticles produced in this way have a poorly defined size, shape and broad size distribution (relative standard deviation $>50 \%$ ), probably due to the poor capping effect of dextran hydroxyl groups, which are attached through hydrogen bonds to the iron oxide particle surface. ${ }^{[39]}$

Samples NF2 and NF3 were obtained using polyol media (Scheme 2) at elevated temperatures $\left(220^{\circ} \mathrm{C} / 12 \mathrm{~h}\right.$ and $200^{\circ} \mathrm{C} / 10$ $h$, respectively) and while NF2 is coated with dextran in a second step, NF3 is in situ coated with citric acid. Figure 1 shows that NF2 is composed of monodisperse $47 \mathrm{~nm}$ nanoflowers with a well defined size (Table 1) and shape. HRTEM images reveal cores of approximately $15 \mathrm{~nm}$ that are densely packed with essentially the same crystal orientation across the entire particle. Similarly, nanoflowers in NF3 are composed of spherical $110 \mathrm{~nm}$ nanoparticles (more than twice that of NF2) with a well-defined size and shape. HRTEM reveals that NF3 consists of very small randomly orientated cores of approximately $4 \mathrm{~nm}$. It has been

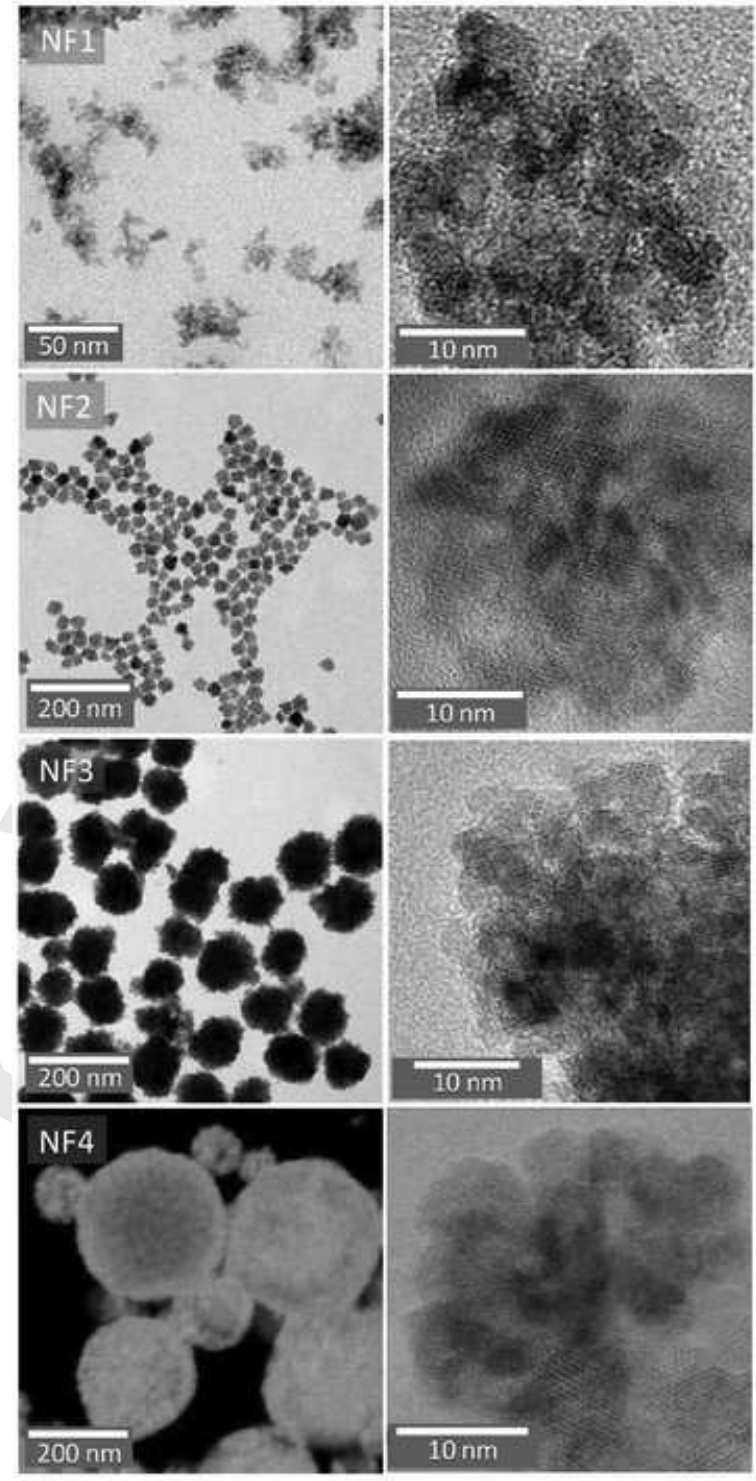

Figure 1. TEM (NF1-NF3), STEM (NF4) (left), and HRTEM images (right) of the different flower-shaped nanoparticles.

reported that polyols play an important role in the reaction and act as solvent, surfactant and reductant. ${ }^{[40]}$ Here, we have used polyols of short chain length (diethylene glycol, DEG, in NF2 and ethylene glycol, EG, in NF3), which enable clustering of the cores to obtain desired multicore structures. For NF2, sodium hydroxide was added to a stoichiometric mixture of iron (II) and iron (III) salts to control the precursor hydrolysis, ${ }^{[41]}$ initiating a burst nucleation followed by the uniform growth of the single cores (LaMer growth). Also, the heating was prolonged for $12 \mathrm{~h}$ to promote clustering and subsequent coalescence of the cores leading to flower-shaped nanoparticles. ${ }^{[42,43]}$ Interestingly, if either the heating time is limited to $2-3 \mathrm{~h}$ under these conditions, or if polyols of longer chain length are used, such as triethylene glycol ${ }^{[44]}$ or 1,2-hexadecanediol, ${ }^{[45]}$ then, only single-core nanoparticles are obtained. Regarding NF3, we observe three key parameters that 
Table 1. Flower-shaped nanoparticles ( $\left.D_{\text {TEM }}\right)$, core $\left(D_{\text {HRTEM }}\right)$, crystal ( $\left.D_{X R D}\right)$ and hydrodynamic $\left(D_{h}\right.$, DLS) sizes estimated through structural characterisation.

\begin{tabular}{|c|c|c|c|c|}
\hline Sample & $\mathrm{D}_{\mathrm{TEM}}(\mathrm{nm})$ & $D_{\text {HRTEM }}(\mathrm{nm})$ & $\mathrm{D}_{\mathrm{XRD}}(\mathrm{nm})$ & $\mathrm{D}_{\mathrm{h}, \mathrm{DLS}}(\mathrm{nm})$ \\
\hline NF1 & $46 \pm 27$ & 7 & $9 \pm 1$ & $192 \pm 84$ \\
\hline NF2 & $47 \pm 17$ & 15 & $15 \pm 1$ & $51 \pm 15$ \\
\hline \multirow[t]{2}{*}{ NF3 } & $110 \pm 13$ & 4 & $8 \pm 1$ & $158 \pm 53$ \\
\hline & $24 \pm 7^{\mathrm{a})}$ & & & \\
\hline NF4 & $172 \pm 70$ & $10^{b)}$ & $10 \pm 1$ & $250 \pm 46$ \\
\hline
\end{tabular}

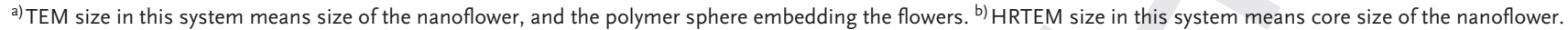

allow the formation of the multi-core structure: the control of temperature and pressure by using an autoclave as reactor, the concentration of the precipitator (sodium acetate, $\mathrm{NaAc}$ ) that promotes the hydrolysis of the $\mathrm{Fe}^{3+}$ ions and thereby controlling the phase transformation and the particle morphology, and finally, the addition of an extra stabilizer (sodium citrate, $\mathrm{Na}_{3} \mathrm{Cit}$ ) that acts as capping agent. High concentrations of NaAc, as those used in NF3, led to flower-shaped nanoparticles composed of uniform cores in size that self-assemble into solid spheres to reduce the surface energy and suffer the so-called recrystallization process. ${ }^{[46]}$ Lower concentrations of NaAc led to the formation of $200 \mathrm{~nm}$ magnetite hollow spheres composed of cores with different sizes. The dissolution of inner small cores and growth of the larger ones on the surface, leads to the formation of such voids that are not observed in this work, by the Ostwald ripening process. ${ }^{[47]} \mathrm{NF} 3$ have such small core size of $4 \mathrm{~nm}$ (Figure 1), due to the presence of sodium citrate $\left(\mathrm{Na}_{3} \mathrm{Cit}\right)$. This extra stabilizer acts as capping agent, significantly suppresses the nuclei growth (in a comparable way to the dextran in NF1 synthesis) and facilitates the final dispersion of the flower-shaped nanoparticles in aqueous media. Carboxyl groups of citric acid coordinate to the iron oxide particle surface via one or two of the carboxylate functionalities, depending upon steric necessity and the curvature of the surface. ${ }^{[48]}$ Alternatively, the use of other additives such as polyacrylic acid (PAA) or polyvinylpyrrolidone (PVP) instead of $\mathrm{Na}_{3} \mathrm{Cit}$ have been reported to account for different particle size (30-250 nm). ${ }^{[47,49,50]}$

As HRTEM images of NF2 and NF3 reveal a different degree of crystallographic orientation texture between the cores (also termed "domains") we have used dark field (DF) imaging analysis to further investigate the nature of the alignment, as is summarized in Figure 2. The bright areas in the DF images contribute to the diffraction spots marked by white arrows in the corresponding selected area electron diffraction (SAED) patterns. In all acquired DF images only part of the nanoflower becomes bright, which clearly illustrates that there is no obvious common crystallographic orientation of the cores. In the case of NF2, for example, we observe that a much larger area becomes bright, indicating orientated assembly of the cores forming the nanoflowers. Interestingly, we observe in the case of NF3 that these domains are much smaller than for NF2.

Finally, in strategy 4 (Scheme 2) nanoflowers were obtained by means of sodium borohydride, which acts as reducing agent and iron (III) acetylacetonate $\left(\mathrm{Fe}(\mathrm{acac})_{3}\right)$, which acts as an iron source. ${ }^{[51,52]}$ In this case we obtain monodisperse $24 \mathrm{~nm}$
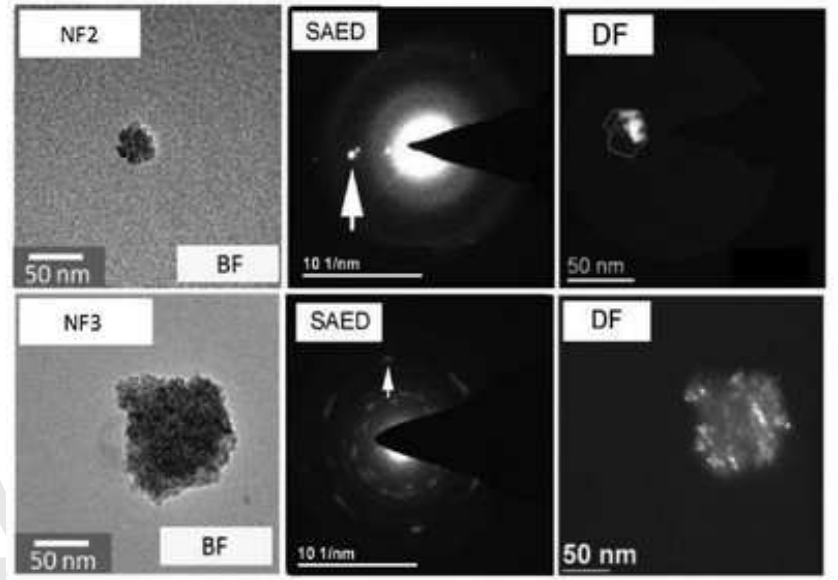

Figure 2. Bright (BF) and Dark Field (DF) analysis of samples NF2 and NF3.

nanoflowers composed of cores of approximately $10 \mathrm{~nm}$ (Figure 1 right). HRTEM imaging suggests that there is a continuous crystallinity through the nanoflower, although contrast differences within a single nanoflower were also clearly visible (Figure S1). For this reduction reaction, it was reported that higher $\mathrm{NaBH}_{4} / \mathrm{Fe}(\mathrm{acac})_{3}$ molar ratios lead to smaller particles. Molar ratios of 10,25 and 40 yielded 8,6 and $5 \mathrm{~nm}$ single-core particles respectively, since $\mathrm{NaBH}_{4}$ increases burst nucleation and decreases the diffusional growth. ${ }^{[52]}$ It was also reported that by elevating the temperature, the crystal size increased. ${ }^{[53]}$ We propose that the key parameter governing the formation of complex nanoflower structures is the molar ratio of $\mathrm{NaBH}_{4}$ to iron precursor; we selected a ratio of around 5, which is relatively low in comparison to other reports. ${ }^{[52]}$ Additionally, the use of prolonged time period (overnight) for the synthesis, also contributes to the formation of this multi-core structure. Nanoflowers synthesized by this route were embedded on polystyrene spheres (Figure S2) via the emulsion solvent evaporation (ESE) process, described in detail elsewhere. ${ }^{[51,52]}$ The final NF4 samples has an average diameter of $172 \mathrm{~nm}$ and a standard deviation of $69 \mathrm{~nm}$, as observed in the STEM image at low magnification (Figure 1). 


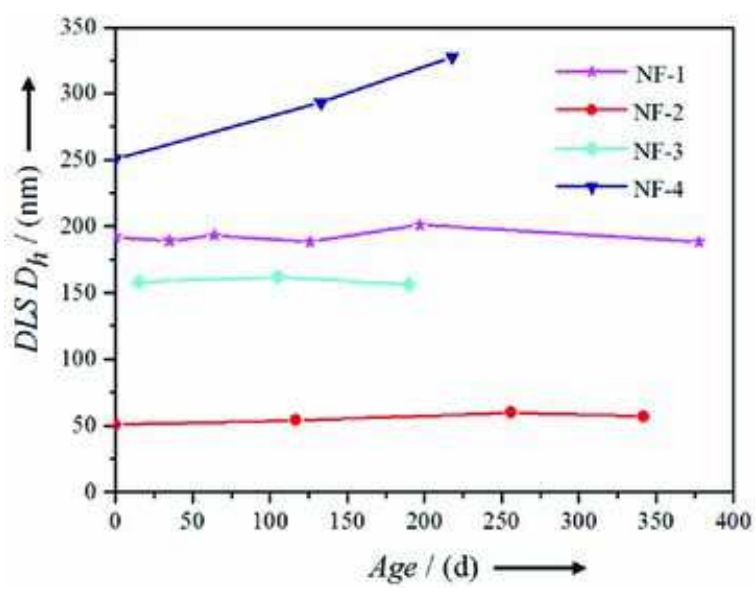

Figure 3. Bright (BF) and Dark Field (DF) analysis of samples NF2 and NF3.

\subsection{Long Term Stability of The Colloids}

Hydrodynamic size $\left(D_{h}\right)$ was measured using dynamic light scattering (DLS) (Table 1). Whilst $\mathrm{D}_{\mathrm{h}}$ varies between $50 \mathrm{~nm}$ (NF2) and $250 \mathrm{~nm}$ (NF4), colloidal stability was maintained for more than 200 days for samples NF1, 2 and 3 (Figure 3). There are mainly two mechanisms for colloidal stability: electrostatic repulsion (if zeta-potential values at a given $\mathrm{pH}$ are higher than 20 $\mathrm{mV}$ or lower than $-20 \mathrm{mV}$ ) and steric repulsion (for the cases where polymeric or macromolecular surfactants and molecules, i.e. dextran or polystyrene coating). NF1 shows a nearly neutral surface $(+2.9 \mathrm{mV})$, whilst NF2 is highly positively charged $(+23$ $\mathrm{mV})$ and NF3 is highly negatively charged $(-40 \mathrm{mV})$. Although NF4 has a surface charge of $-62 \mathrm{mV}$ we observe an increase in its hydrodynamic size overtime (Figure 3), which is probably due to aggregation phenomena because of its large particle size.

\subsection{Core Arrangement within the Nanoflower and Interparticle Interactions}

The crystal structure of all nanoflowers was identified as a mixture of magnetite $\left(\mathrm{Fe}_{3} \mathrm{O}_{4}\right)$ and maghemite $\left(\gamma-\mathrm{Fe}_{2} \mathrm{O}_{3}\right)$ using $\mathrm{X}$ ray diffraction (XRD) and ${ }^{57} \mathrm{Fe}$ Mössbauer spectroscopy (Figure 4 and 5, respectively). The acquired XRD data were analyzed through the Rietveld refinement method (Figure S3). All the reflections were accounted with the $F d-3 m$ space group with no sign of any spurious phase within the $(<5 \%)$ uncertainty inherent in XRD. Calculated values of lattice parameters are in the range of 8.35-8.37 $\AA$, which lie between the bulk value reported $\sim 8.39 \AA$ and $\sim 8.34 \AA$ for magnetite ${ }^{[54]}$ and maghemite, ${ }^{[55]}$ respectively (Table S1 and S2). This indicates that all samples are at least partially oxidized to maghemite; this is in particularly notable for NF2, which was subjected to an oxidation treatment with iron nitrate prior to dextran coating. Bulk magnetite and maghemite can be distinguished by their different lattice parameter, but in nanosized materials, the combination of peak broadening and variation of the lattice parameters make this challenging. In addition, whereas the space group of magnetite is well established to be $F d-3 m$, maghemite may be present in different space groups depending of the vacancy ordering, $\mathrm{Fd}$ -

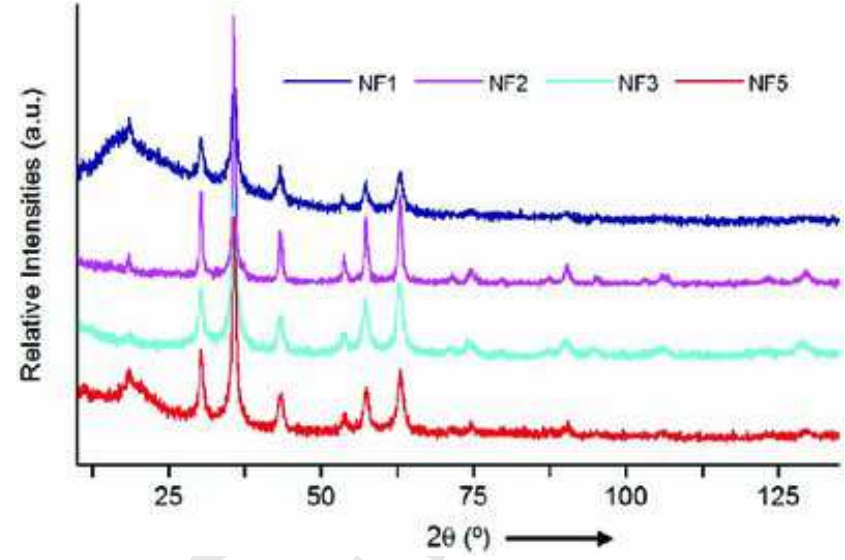

Figure 4. Room-temperature $X$-ray diffraction patterns of the different flower-shaped nanoparticles. The hump observed at low angles (below $25^{\circ}$ ) in NF1 and NF4 samples results from their amorphous organic content.

$3 m$ being one of the possibilities. ${ }^{[56]}$ The crystal sizes, previously described (Scheme 1), were calculated from the XRD data and are summarized in Table 1, along with core sizes measured by HRTEM (the term core is likewise described in Scheme 1). XRD sizes range from 8 (1) to 15 (1) $\mathrm{nm}$. Differences in terms of crystal size allow us to categorize the nanoflowers into three regimes, that is below, equal or larger than $10 \mathrm{~nm}$. NF3 has the largest nanoflower size $(110 \mathrm{~nm})$ and is composed of crystals of less than $8 \mathrm{~nm}$, whereas NF2 with much smaller nanoflowers (47 nm), has crystals of $15 \mathrm{~nm}$, the largest in this series of nanoflowers. These results are in very good agreement with the observations in both, HRTEM and DF images. Lastly, both NF1 and NF4 are intermediate systems with crystal sizes of 9 and 10 $\mathrm{nm}$, respectively.

${ }^{57} \mathrm{Fe}$ Mössbauer spectroscopy measurements highlight their crystalline nature and shed light on the influence of the size and structural arrangement of the cores on the magnetic properties. Room temperature (RT) spectra are shown in Figure 5. All four samples comprise magnetically split spectra but with different degrees of magnetic relaxation due to their crystallinity (seen as line broadening, and partially collapsed or 'hanging' sextets). In all four spectra, more than half of the spectral area is magnetically split and we surmise that, despite such relaxation effects, the samples are all below their superparamagnetic blocking temperature at room temperature on the time scale of Mössbauer spectroscopy ( $1 \mathrm{~ns})$. Magnetic relaxation effects are most pronounced in the NF1-spectrum (Figure 5a), which is a superposition of a superparamagnetic doublet (13\%) and a sextet with broad lines. Relaxation effects are least pronounced in the NF2-spectrum (Figure 5b), which features a sextet with relatively sharp lines. It is noticeable that samples NF1 and NF2, which have similar TEM particle sizes, show very different magnetic relaxation. The longer magnetic relaxation times of NF2 are likely to be a consequence of the core arrangement within the nanoflowers (showing crystalline correlation length of ca. XRD size $=15 \mathrm{~nm})$ compared to NF1 $(9 \mathrm{~nm})$ and is in agreement with previous observations that exchange interaction between surface atoms of aggregated nano-scale cores tends to 

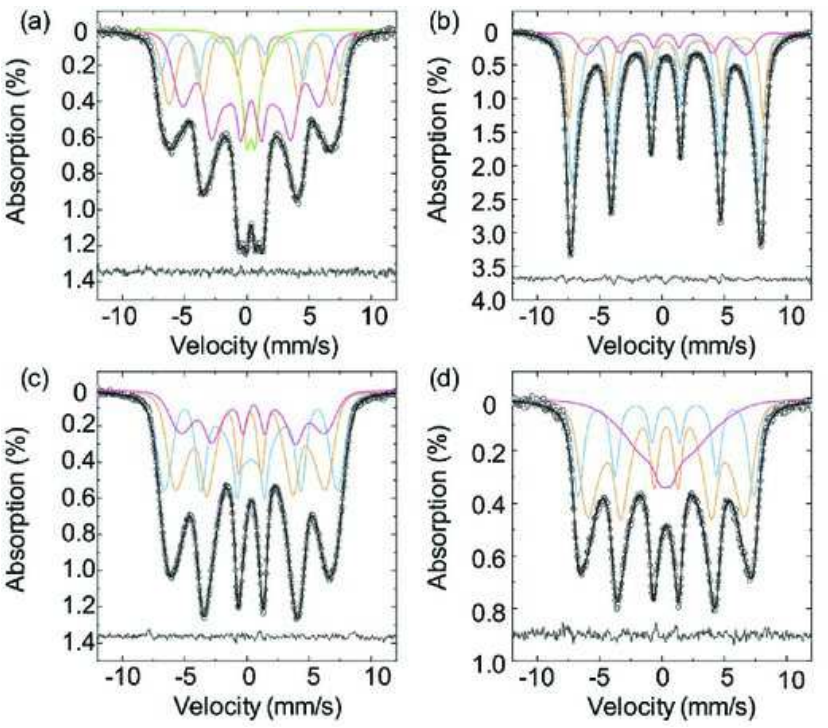

Figure 5. Room-temperature ${ }^{57} \mathrm{Fe}$ Mossbauer Spectroscopy spectra of: (a) NF1, (b) NF2, (c) NF3 and (d) NF4. All spectra have been fitted according to Fock et al 2016 using Voigitan line shapes. Total fit is shown by solid black line (Voigtian sub-spectra in grey). The quality of each fit is indicated by the residual - the difference between observed and fitted intensities - shown beneath each spectrum.

suppress superparamagnetic relaxation. ${ }^{[56]}$ NF3 and NF4 display almost identical relaxation (Figure $5 c-d$ ), which is especially interesting as they are comprised of quite different core and nanoflower sizes. This suggests the presence of much stronger interactions between the cores in the particle in NF3 compared to the larger polystyrene-embedded NF4 nanoflowers. Furthermore, the smaller cores in NF3 seem to interact more strongly than the larger cores in NF1. These results perfectly match our previous observations by HRTEM of cores densely packed in a solid sphere in NF3, compared to the loosely packed cores in NF1 (Figure 1). RT Mössbauer spectra support the hypothesis that flower-shaped nanoparticles of larger core size show reduced relaxation, and that an increased density of cores within the nanoflowers suppresses magnetic relaxation.

The mean isomer shift ("centre") of the Mössbauer spectra relative to $\alpha$-Fe is sensitive to the oxidation state of iron and allows for the quantification of the magnetite-maghemite content in the samples via the method described by Fock \& Bogart et al. ${ }^{[57]}$ The method is applicable to room-temperature spectra, but in case of spectra obtained at low temperature (18 and 80 K) (see Figure S4), where relaxation effects are negligible and hence spectral lines sharper, the mean isomer shift can be obtained with less uncertainty (see Table S3). ${ }^{[57]}$ From this, we find that the percentages of $\mathrm{Fe}$ atoms in the form of magnetite in the samples are, $9 \pm 2,5 \pm 3,5 \pm 2$, and $3 \pm 2$ for NF1, NF2, NF3 and NF4 respectively, i.e. the samples are essentially maghemite. This means that the differences found in XRD lattice parameters are related to differences in the degree of structural order rather than to variation in magnetite/maghemite content. Indeed, the largest lattice parameters correspond to NF1 and NF3, which also have the smallest core sizes and therefore larger fraction of surface atoms. Samples NF1 and NF3 were synthesized in the presence of dextran and citric acid that absorb on the core surface hampering the contact between adjacent cores.

The static magnetic properties were evaluated for all samples in liquid suspensions by DC magnetometry measurements at $300 \mathrm{~K}$ (Figure 6a). The effective magnetic size of the nanoflowers was obtained by modeling the $\mathrm{M}(\mathrm{H})$ curve using the classical superparamagnetic Langevin function (see SI). These results are presented in Table 2. The magnetization curve of NF3 was fitted by a monomodal size distribution indicating that the cores have a uniform size with a single effective magnetic diameter of $16 \mathrm{~nm}$. In the case of NF2, we observe a better fit to the experimentally measured $\mathrm{M}(\mathrm{H})$ curve when our Langevin fit uses a bimodal size distribution. This gives two effective magnetic sizes; the first size of $25 \mathrm{~nm}$ has a very narrow size distribution of 0.1 while the second distribution has a much smaller size of $2 \mathrm{~nm}$. In addition, this sample has high saturation magnetization value and high initial susceptibility, both of which are due to the high fraction of particles with large magnetic sizes. Likewise, for NF1 and NF4, the $\mathrm{M}(\mathrm{H})$ curves are better fitted with a bimodal size distribution. Both samples consist magnetically of two particle fractions with different magnetic diameters although inspection of the initial magnetization slope at low fields suggests that NF4 contains a larger fraction of larger sizes, which justifies the larger saturation magnetization. It should be noted that in many cases the derived effective magnetic sizes cannot directly be compared with the particle size determined by TEM or the crystal sizes determined by XRD.

The effective magnetic size modeled by the Langevin function is further a distribution of magnetic moments projected on a sphere. This sphere is usually smaller than the TEM size due to the surface dead layer. ${ }^{[58]}$ For the special case of flower-shaped nanoparticles consisting of packed cores forming a multi-core structure, this sphere is smaller than the flower TEM sizes but larger than the core size for all the systems in this work. The interactions between the cores can lead to magnetic sizes that are larger than the crystal sizes measured by XRD. DC magnetometry data indicates that the nanoflowers are, in general, better fitted using a bimodal size distribution and indicates that size and shape of the cores varies across the nanoflower, as revealed by DF images (Figure 2). However, for NF3, whose cores are smaller and randomly distributed, the best fit of the magnetization curve is with a monomodal distribution.

Dynamic magnetic properties of the nanoflowers were characterized by AC susceptibility (ACS) vs frequency and temperature. These measurements were conducted on samples in both dispersed and immobilized states (See Figure 6b and SI). An ACS hydrodynamic diameter was calculated using the generalized Debye model (ACS $\left.D_{\mathrm{h}, 1}\right)^{[59]}$ and the multi-core extended model $\left(\mathrm{ACS}_{\mathrm{h}, 2}\right)^{[60]}$ as summarized in Table 2. NF1 shows a weak maximum of the imaginary part at $\sim 80 \mathrm{~Hz}$ caused by Brownian rotation. The gradual decay of the real part of both dispersed and immobilized particles (Figure S5) indicates a wide distribution of relaxation times. This is further supported by the constant imaginary part of the sample with immobilized nanoparticles, which is superimposed by the weak Brownian relaxation peak for the dispersed sample, which indicates that the majority of particles relax via the internal Néel mechanism. Using the generalized Debye model we obtain an average hydrodynamic diameter of $251 \mathrm{~nm}$ whilst this value decreases to 
Table 2. Parameters determined through magnetic characterisation: Saturation magnetisation (Ms) and magnetic size $\left(d_{c, 1}\right.$ and $\left.d_{c, 2}\right)$ by $D C$ Magnetometrya), Hydrodynamic size (ACS $D_{h, 1}$ and ACS $D_{h, 2}$ ) by ACS versus frequency (Debye model and Multi-Core extended model, respectively) ${ }^{\text {b) }}$ and anisotropy constant $\left(\mathrm{K}_{\mathrm{ACvT}} \mathrm{T}\right.$ by $\mathrm{ACS}$ versus temperature. ${ }^{\mathrm{C})}$

\begin{tabular}{lccccccc}
\hline Sample & $\begin{array}{l}\mathrm{M}_{\mathrm{s}} /\left(\mathrm{Am}^{2} / \mathrm{kg} \mathrm{Fe}\right) \\
(\sigma)\end{array}$ & $\mathrm{d}_{\mathrm{c}, 1} /(\mathrm{nm})(\sigma)$ & $\mathrm{d}_{\mathrm{c}, 2} /(\mathrm{nm})(\sigma)$ & $\beta^{[\mathrm{d}]}$ & $\mathrm{ACS} \mathrm{D}_{\mathrm{h}, 1} /(\mathrm{nm})(\sigma)$ & $\mathrm{ACS} \mathrm{D}_{\mathrm{h}, 2} /(\mathrm{nm})$ & $\mathrm{K}_{\mathrm{ACVT}} /\left(\times 10^{4} \mathrm{~J} / \mathrm{m}^{3}\right)$ \\
\hline NF1 & $93(3)$ & $4.3(0.52)$ & $18.7(0.24)$ & 0.44 & $251(116)$ & 180 & $2.5(2)$ \\
NF2 & $112(3)$ & $2(0.8)$ & $24.7(0.08)$ & 0.74 & $54(11)$ & 45 & $2.3(2)$ \\
NF3 & $103(3)$ & - & $16(0.1)$ & - & $247(5)$ & 175 & $1.8(2)$ \\
NF4 & $119(4)$ & $2.5(0.56)$ & $13.6(0.03)$ & 0.69 & - & - & $4.0(3)$ \\
\hline
\end{tabular}

a) Measurements performed in the dried samples (powder form). ${ }^{\text {b) }}$ Measurements performed in both colloidal dispersion and immobilized samples. ${ }^{\text {c) }}$ Measurements performed in the colloidal dispersion. ${ }^{d)} \beta$ is the normalized fraction of the larger particle size distribution.
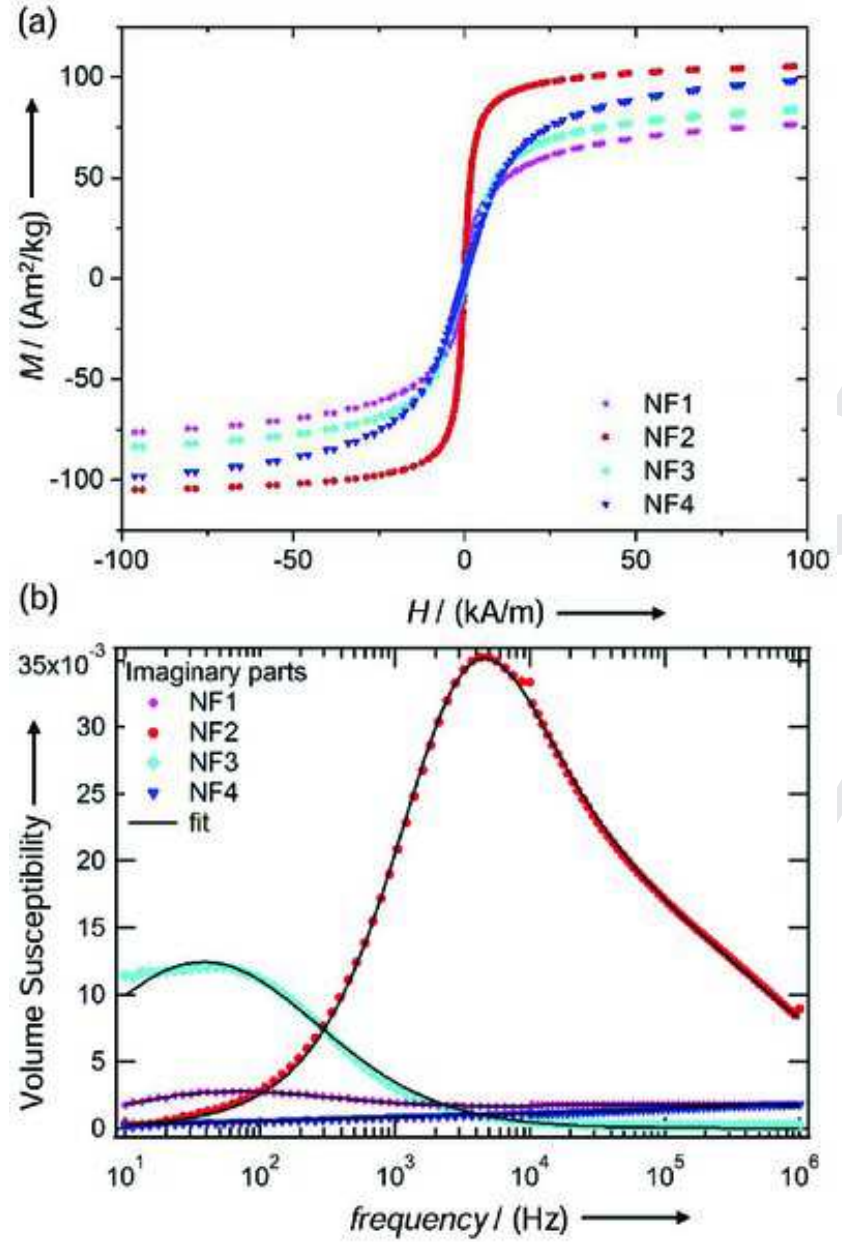

Figure 6. Magnetic characterization at $300 \mathrm{~K}$. (a) DC Magnetization curves and (b) AC susceptibility vs frequency (imaginary part) of all flower-shaped nanoparticles in suspension (The solid line shows best fit with generalized Debye model).

$180 \mathrm{~nm}$ using the multi-core extended model, which is comparable to the value measured by DLS (192 nm). However, for NF2, a pronounced peak in the imaginary part at $5 \mathrm{kHz}$ clearly indicates Brownian rotation with a Néel contribution of 10-20\%. The linear decay of the real part of the immobilized sample when plotted vs. $\ln (\mathrm{f})$ indicates a wide distribution of Néel relaxation times, i.e. anisotropy energies. ${ }^{[61]}$ Modeling these data using the generalized Debye model yields a mean hydrodynamic diameter of $54 \mathrm{~nm}$ and $45 \mathrm{~nm}$ using the Debye and multi-core extended model, respectively, both of which are in good agreement with the value measured in DLS measurements $(51 \mathrm{~nm})$. For NF3, the Brownian relaxation peak in the out-of-phase component of the AC susceptibility is at about $50 \mathrm{~Hz}$ with a shallow shoulder toward low frequencies indicating agglomeration. The low frequency relaxation disappears when the nanoparticles are diluted. The real part levels off at high frequencies together with a nearly zero imaginary part which can be caused by intra-potential-well contributions $^{[62-64]}$ and by nanoparticles that follow the excitation field via the internal Néel mechanism with relaxation times well below $1 \mu$ s, i.e. moments can follow the sinusoidal excitation field without phase lag up to at least $1 \mathrm{MHz}$ frequency. In this case, the values of average hydrodynamic diameters are $247 \mathrm{~nm}$ using the generalized Debye model and $175 \mathrm{~nm}$ using the multi-core model, the latter being more comparable to the value measured by DLS (158 nm). Interestingly, for NF4, we observed almost no difference between immobilized and dispersed particles (Figure S5), which is typical for a nanoparticle system that undergoes 100\% Néel relaxation. The Néel relaxation peak in the out-of-phase component is in the range of several $\mathrm{MHz}$, i.e. outside the measurement window: since the MNP dynamics are dominated by the Néel mechanism, no information can be obtained on their hydrodynamic size.

ACS vs temperature was performed at low temperature (5$260 \mathrm{~K})$ to derive an effective anisotropy constant for the nanoflowers that is presented in Table 2 . The in-phase component $\left(\chi^{\prime}\right)$ of the AC susceptibility approaches a frequency independent value as $T \rightarrow 0$, corresponding to the intra-potential-well response of the particle moments. ${ }^{[64]}$ Using the low-T $\chi^{\prime}$-values, we determined the effective anisotropy constant values (K) of: 2.5 (2), 2.3 (2), $1.8(2)$ and $4.0(3)\left(\times 10^{4} \mathrm{~J} / \mathrm{m}^{3}\right)$ for NF1, 2, 3 and 4 respectively (see Figure S5 and Table S6 in SI for details). Interestingly, we find that the nanoflower system with the largest anisotropy constant (NF4), is also the only system for which blocking temperature is close to room temperature (see Figure S5 b). This implies that the embedded nanoflowers might also display some order on the poly (styrene) sphere, as described for some magnetic nanocrystals forming close-packed structures, which showed an increase in anisotropy. ${ }^{[65]}$ Conversely, NF1-3 

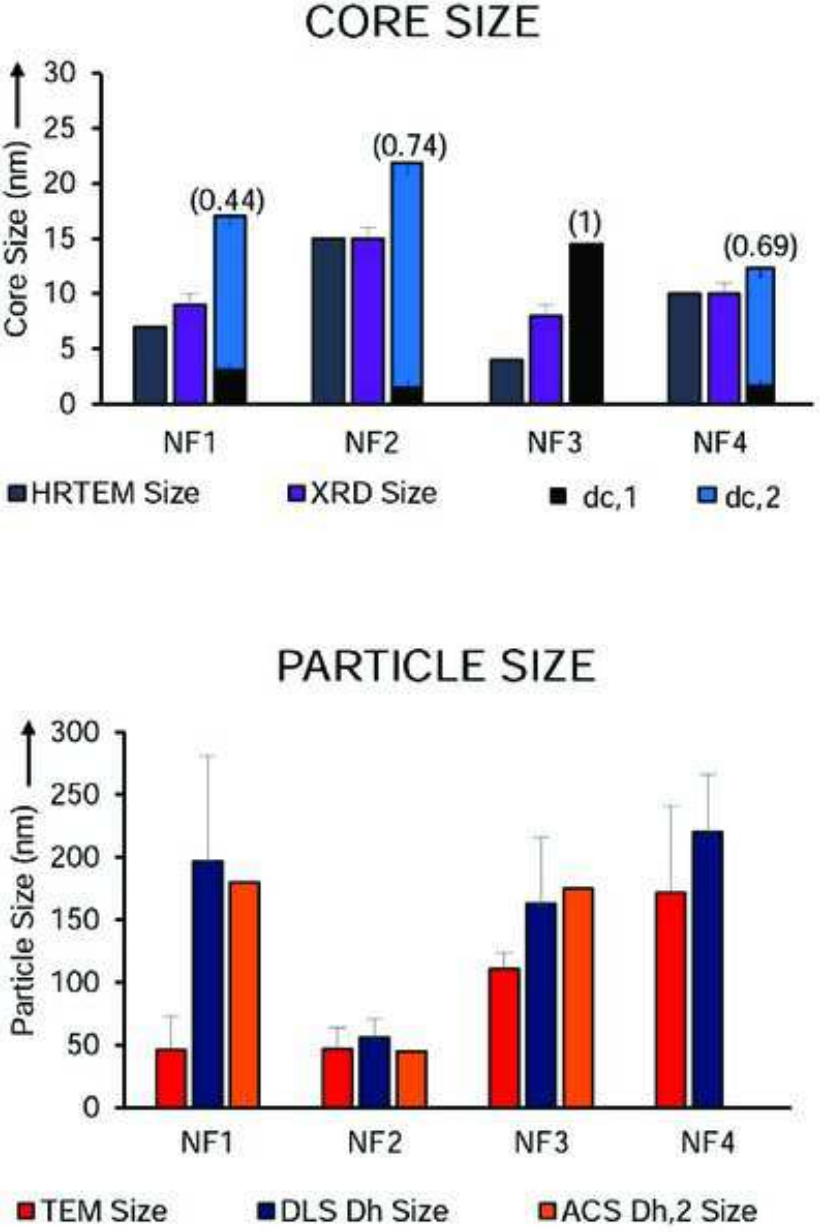

Figure 7. Core (above) and particle size (below) of the flower-shaped nanoparticles as determined by structural and magnetic means, according to Table 1 and 2. Between brackets, $\beta$ is included, the normalized fraction of the larger particle size distribution as determined by DC magnetometry.

are clearly blocked in the temperature range $5-390 \mathrm{~K}$ for $\mathrm{f}<$ $1000 \mathrm{~Hz}$.

\subsection{Description of the Structure of a Nanoflower}

The results obtained for the core and particle size for each flowershaped system through different characterization techniques are summarised in Figure 7. The size of the cores range from 4 to $15 \mathrm{~nm}$ and the size of the particle range from 50 to $250 \mathrm{~nm}$. Differences in both, core and particle size reflect the complicated nature of these materials. In general, the size calculated via $\mathrm{XRD}\left(\mathrm{D}_{\mathrm{XRD}}\right)$ is larger than that estimated via HRTEM ( $\left.\mathrm{D}_{\text {HRTEM }}\right)$. This reflects the multi-core nature of the samples, whose cores are fused to a greater or lesser degree to form flower-shaped structures.

In those cases with high values of $\mathrm{D}_{\mathrm{XRD}} \approx \mathrm{D}_{\text {HRTEM }}>10 \mathrm{~nm}$ (NF2 and NF4), significant coalescence of the cores is detected, which justifies the highest $\mathrm{M}_{\mathrm{s}}$ values displayed by those samples. For all cases, the magnetic size deduced from our Langevin based modeling $\left(\mathrm{d}_{\mathrm{c}, 2}\right)$ is larger than both DHRTEM and DXRD suggesting the presence of interactions between cores and thus shedding light on the collective behaviour within the nanoflowers. For samples that possess larger effective magnetic size, such as NF2, we observe both an increase in $\chi_{0}$ in the RT magnetization curve and an increase in magnetic relaxation times, as demonstrated by a ${ }^{57} \mathrm{Fe}$ Mössbauer spectrum sextet with relatively sharp lines at RT. Samples with a more reduced effective magnetic size (NF1, NF3 and NF4) have a visibly smaller $\chi_{0}$ in the magnetization curve and also have reduced relaxation, i.e. less Mössbauer spectral area within the sextet. We surmise that when there is a close contact between cores within a particle, the continuity of the crystal orientation is ensured favouring magnetic ordering across the interfaces. This is indeed the case of the structures previously called nanoflower that showed one order of magnitude higher heating rates than the specific absorption rate (SAR) reported for conventional $11 \mathrm{~nm}$ maghemite nanoparticles in the same field exposure conditions. ${ }^{[42]}$

Regarding the particle size, samples in which DLS $\mathrm{D}_{\mathrm{h}} \gg$ $\mathrm{D}_{\text {TEM }}$ either contain a large amount of coating and water hydration on their surface or have collective behaviour between the particles (interparticle interactions, i.e. dipolar interactions). The former can be detected by IR spectroscopy and quantified by thermogravimetric analysis (See Figure S7). This is the case of NF1 and NF2 that have similar $D_{\text {TEM }}$ particles sizes and therefore similar surface-area-to-volume ratios but they have very different organic content ( $80 \%$ for NF1 and $20 \%$ for NF2). Consequently, DLS $D_{h}$ size of NF1 is much larger than $D_{\text {TEM }}$ and hence indicates a larger amount of dextran present on the nanoparticles surface compared to NF2. The existence of organic component is already supported by the XRD data displayed in Figure 4, where the hump that appears for NF1 at low angles (below $25^{\circ}$ ) is consequence of such contribution.

The collective behaviour that is related to interparticle interactions can be detected by AC susceptibility. All samples have comparable hydrodynamic sizes obtained by both DLS $\mathrm{D}_{\mathrm{h}}$ and ACS $\mathrm{D}_{\mathrm{h}, 2}$ (modeled by the extended multi-core function), confirming that dipolar interactions between particles are minimized in these colloids and explaining the observed long-term stability. In the case of NF3, dipolar interactions between particles may cause the formation of chains and explain the observed change in AC spectrum with sample dilution.

Comparing core and particle size from the magnetic measurements, i.e. effective magnetic size $\left(\mathrm{d}_{c, 2}\right)$ and hydrodynamic size ACS $D_{h, 2}$, we have identified three different flower-shaped multi-core systems. For NF2, the effective magnetic size tends to approach the nanoflower size (ACS $D_{h, 2} / d c, 2 \sim 1.8$ ) indicating strong magnetic interactions between cores within a particle. Cores inside the nanoflower are not only oriented but also in close contact leading to exchange interactions, and therefore collective magnetic behaviour. For NF1 and NF3, the effective magnetic size is much smaller than the flower size (ACS $\mathrm{D}_{\mathrm{h}, 2} / \mathrm{d}_{\mathrm{c}, 2} \sim 10$ ) indicating less contact between cores, which in NF1 may be loosely packed within the flower. Much more complicated is the case of NF4, where nanoflowers were embedded onto polymeric spheres creating a superstructure. The relatively small nanoflower size, high anisotropy constant, but low blocking temperature of NF4 suggests that the finite size of 
the nanoflower systems is one of the key parameters that dictates their Néel relaxation properties.

\subsection{Toward the Standardization: Comparison of the Nanoflower Synthesis}

Differences between the systems (NF1-4) are directly related to the various synthetic routes (Strategy 1-4), in terms of formation mechanism (clustering and coalescence, clustering and recrystallization or Ostwald ripening) and free energy involved in the process and experimental conditions ( $\mathrm{pH}$, surface charge, temperature, pressure and time). Table 3 provides a comparison of the key parameters determining the core assembly and their implication on the resulting unique colloidal and magnetic properties. Additionally, from the synthesis standardization point of view, the advantages and disadvantages of each strategy are highlighted. NF1 offers the possibility of large production and allows the synthesis of larger batches in aqueous media. The nanoflowers are directly stabilized by the presence of dextran in the reaction media and present long-term stability for at least 500 days (Figure 3). The use of polyol media provides great control over core aggregation, which yields multi-core structures that consist of cores in more or less contact (NF2 and NF3) depending on the synthesis conditions. Furthermore, polyol media allows the use of higher reaction temperatures $\left(200^{\circ} \mathrm{C}\right)$ leading to fused cores within a particle e.g. NF2. However, in NF3, the use of an extra additive (sodium citrate) to facilitate the final dispersion of the particles in aqueous media hampers the aggregation of the cores in a similar way to dextran in the synthesis of NF1. Aqueous suspensions of these systems present long-term stability for at least 200 days (Figure 3). However, it should be taken into account that high temperatures over long time as applied in the synthesis of NF2 and NF3 (10-12 h) generally lead to high-energy consumption (costs). Moreover, one-pot syntheses such as those followed to obtain NF1 and NF3 have advantages compared to the conventional step-wise reactions as used for NF2 and NF4.

\section{Conclusion}

Analysis of the self-assembly process conducted in this work leads to the formation of flower-shaped nanoparticles with properties and possible applications that differ strongly from singlecore particles of similar size and corresponding bulk material. Synthesis reagents and experimental conditions are key factors to control the core and particle sizes, as well as intra- and interparticle interactions, i.e. between cores and particles in suspensions. The results obtained from different characterisation techniques have been brought together to obtain a self-consistent picture that describes how structural and magnetic properties are interrelated in those systems. Controlling self-assembly of magnetic multi-core nanoparticles allow the design of optimal magnetic properties of the colloids as a function of its specific application.

\section{Experimental Section}

Synthesis of Flower-Shaped Nanoparticles-Sample NF1: Dextran coated iron oxide nanoparticles were synthesized by partial oxidation of $\mathrm{Fe}(\mathrm{OH})_{2}$ and green rust. Typically, $\mathrm{KNO}_{3} 2 \mathrm{M}, \mathrm{NaOH}$ $2 \mathrm{M}$ and dextran solution ( $5 \mathrm{~mL}$ ) were added to MilliQ water (25 $\mathrm{mL}$ ) and degassed by bubbling with nitrogen. ${ }^{[66]}$ Under stirring, $\mathrm{FeSO}_{4} \cdot 7 \mathrm{H}_{2} \mathrm{O} 0.3 \mathrm{M}(8.3 \mathrm{~mL})$ were slowly added to the initial solution resulting in the precipitation of gel-like $\mathrm{Fe}(\mathrm{OH})_{2}$ and green rust. To accelerate the oxidation process the solution was heated to $90{ }^{\circ} \mathrm{C}$ for 4 hours. Finally, the particles were purified by dialysis and centrifugation resulting in a stable colloidal dispersion.

Synthesis of Flower-Shaped Nanoparticles-Sample NF2: Dextran coated $\gamma-\mathrm{Fe}_{2} \mathrm{O}_{3}$ NPs were synthesized by a polyol method adapted from Lartigue et al. ${ }^{[24]}$ In a typical procedure of $\mathrm{FeCl}_{3} \cdot 6 \mathrm{H}_{2} \mathrm{O}(4 \mathrm{mmol})$ and $\mathrm{FeCl}_{2} \cdot 4 \mathrm{H}_{2} \mathrm{O}(2 \mathrm{mmol})$ were dissolved in diethylene glycol (DEG) and N-methyldiethanolamine (NMDEA) (1:1 v/v, $80 \mathrm{~g})$ at room temperature. The solution was stirred for $1 \mathrm{~h}$. Separately, $\mathrm{NaOH}(16 \mathrm{mmol})$ was dissolved in the polyol mixture $(40 \mathrm{~g})$ and subsequently added to the iron chlorides solution. The mixture was then stirred for 3 hours at room temperature. The temperature of the solution was then elevated to $210^{\circ} \mathrm{C}$ using a ramp temperature of $2{ }^{\circ} \mathrm{C} / \mathrm{min}$. Once the temperature reached $210^{\circ} \mathrm{C}$, the solution was stirred for 12 $\mathrm{h}$ at the same conditions. Then the mixture was cooled down to room temperature. The nanoparticles were separated magnetically and washed four times with a mixture of ethanol and ethyl acetate $(1: 1, \mathrm{v} / \mathrm{v})$ and once with $10 \%$ nitric acid. Then, a, aqueous solution $(20 \mathrm{~mL})$ containing iron (III) nitrate $(8.25 \mathrm{~g})$ was added to the NPs, and the mixture was heated to $80^{\circ} \mathrm{C}$ for 45 minutes. After cooling down to room temperature, the nanoparticles were washed again, once with $10 \%$ nitric acid, once with ethanol and once with acetone. The sediment was dried for $2 \mathrm{~h}$ at $40^{\circ} \mathrm{C}$. Finally, the particles were re-dispersed in water $(20 \mathrm{~mL})$. Coating with dextran was carried out by high-pressure homogenization process. ${ }^{[67]}$

Synthesis of Flower-Shaped Nanoparticles-Sample NF3: $\gamma$ $\mathrm{Fe}_{2} \mathrm{O}_{3}$ NPs were synthesized by a polyol-mediated method previously reported by Liu J et al. ${ }^{[46]}$ Typically, iron chloride $(2.702$ g) was dissolved in ethylene glycol (EG, $47 \mathrm{~mL}$ ) under magnetic stirring. Then trisodium citrate $\left(\mathrm{Na}_{3} \mathrm{Cit}\right)$ and sodium acetate (NaAc) was gradually added under mild heating and magnetic stirring. The final concentration of the reagents is: $\left[\mathrm{FeCl}_{3}\right]=$ $0.21 \mathrm{M},\left[\mathrm{Na}_{3} \mathrm{Cit}\right]=0.05 \mathrm{M}$, and $[\mathrm{NaAc}]=0.76 \mathrm{M}$. The mixture was stirred vigorously for $30 \mathrm{~min}$ and then sealed in a Teflonlined Aluminium autoclave (125 mL capacity) and maintained at $200{ }^{\circ} \mathrm{C}$ for $10 \mathrm{~h}$. After cooling down to room temperature, the black product was washed 3 times with distilled water by centrifugation.

Synthesis of Flower-Shaped Nanoparticles-Sample NF4: Iron oxide nanoparticles were produced by a reduction method that was based on reports in the literature. ${ }^{[51,52]}$ In brief, iron acetylacetonate $(9.43 \mathrm{~g})$ and sodium borohydride $(5.04 \mathrm{~g})$ were added to a mixture of $\mathrm{H}_{2} \mathrm{O}$ and THF $(400 \mathrm{~mL})$, as detailed in the literature report. The mixture was stirred overnight at $150 \mathrm{rpm}$. The black precipitates were collected by centrifugation and rinsed with deionized water. A solution consisting oleic acid $(67 \mathrm{~mL})$ 
Table 3. Comparison resulting from the analysis of the synthesis and characterization of the flower-shaped iron oxide nanoparticles.

\begin{tabular}{|c|c|c|c|c|c|}
\hline General comparison & & NF1 & NF2 & NF3 & NF4 \\
\hline \multirow[t]{3}{*}{ Synthesis strategies } & Reaction & One step & Multistep & One step & Multistep \\
\hline & Production Yield & Large & Moderate & Moderate & Low \\
\hline & Mechanism & Clustering & $\begin{array}{c}\text { Clustering }+ \\
\text { Recrystallization }\end{array}$ & Clustering + Coalescence & $\begin{array}{c}\text { Clustering }+ \text { Coalescence }+ \\
\text { Embedment }\end{array}$ \\
\hline \multirow{2}{*}{$\begin{array}{l}\text { Colloids Flower-shaped } \\
\text { magnetic nanoparticles }\end{array}$} & Interaction between cores & Low & High & Moderate-high & Moderate \\
\hline & $\begin{array}{l}\text { Interaction between } \\
\text { particles }\end{array}$ & Low & Low & Moderate & High \\
\hline Advantages & & Long term colloidal stability & $\begin{array}{c}\text { Large saturation } \\
\text { magnetization and large } \\
\text { susceptibility }\end{array}$ & $\begin{array}{l}\text { Large magnetic moment } \\
\text { per particle }\end{array}$ & $\begin{array}{l}\text { Magnetic superstructure } \\
\text { with high anisotropy }\end{array}$ \\
\hline Disadvantages & & $\begin{array}{l}\text { Wide particle size } \\
\text { distribution }\end{array}$ & High energy con & suming synthesis & $\begin{array}{l}\text { Reduced long term colloidal } \\
\text { stability }\end{array}$ \\
\hline
\end{tabular}

in THF $(533 \mathrm{~mL})$ was added to the suspension and shaken for 2 hours. The nanoparticles were precipitated with ethanol and collected by centrifugation, followed by redispersion in chloroform $(10 \mathrm{~mL})$. In solution 1 , the particle solution $(6.25 \mathrm{ml})$ was added gently to chloroform $(20.25 \mathrm{~g})$. In the case of sample NF4, poly (styrene) (500 mg) is previously dissolved in the chloroform) in a $25 \mathrm{ml}$ glass vial; In solution 2, poly (styrene-alt-maleic acid)sodium salt solution (13.96 g) and sodium lauryl sulphate (3.35 g) solution is added to DIW (1 L). Solution 1 is added to solution $2(53 \mathrm{~mL})$ in a $100 \mathrm{~mL}$ Erlenmeyer flask by ultra sonication for 15 min with ultrasonic probe Amplitude $40 \%$ in an ice bath. Chloroform was evaporated under reduced pressure. The solution is centrifuged $2000 \mathrm{rpm}$ for $5 \mathrm{~min}$. The supernatant is collected and the precipitate is discarded.

Characterization-TEM Analysis: A FEI Tecani G2 T20 transmission electron microscope (TEM) equipped with $\mathrm{LaB}_{6}$ electron gun and operated at $200 \mathrm{kV}$ was used in this study. TEM sample preparation was done by putting a droplet of the diluted suspension in water on holey carbon film coated TEM Cu grid, and then letting it dry in air at room temperature. Images were acquired in bright field (BF) imaging mode and evaluated using DigtalMicrograph software. The method used for measuring the diameter of both the multicore particles and iron oxide nanocrystals is described as follows. First, a circle was drawn on the image in DigitalMicrograph. Then the circle was adjusted so that its size is as small as possible but still covers a single particle or nanocrystal to be measured. The diameter of this circle is subsequently taken as the diameter of the particle. Structure of individual flower particles were also analysed by selected area electron diffraction (SAED), bright field and dark field (DF) imaging and high resolution transmission electron microscopy (HRTEM) imaging.

Characterization-X-Ray Powder Diffraction Analysis: Chemical composition and core crystal structure were confirmed along with the determination of crystal size by X-ray powder diffraction (XRD) experiments performed at room temperature (RT) in a D8 Advance diffractometer, using a $\mathrm{Cu} \mathrm{K} \alpha$ radiation with Bragg-Brentano geometry. The samples in solution were freezedried for the subsequent XRD measurement, which were placed on a Si single-crystal low background sample holder that was rotated at $15 \mathrm{rpm}$ to improve random orientation of crystallites, while minimizing the effect of preferred orientations within the sample. The acquired data were analysed through the Rietveld refinement method using the FullProf Suite software. ${ }^{[68]}$ To describe the peak profiles, a Thompson-Cox-Hastings function was chosen to guarantee a good description of the width excess to extract the average crystal size (D) of the samples. The figures of merit are acceptable for fine particle systems. To fully account for peak broadening, it is necessary to include some lattice strain contribution $(\varepsilon)$. These $\varepsilon$ values lie around $500 / 000$ and it is a sign of crystal defects, especially on particle surfaces.

Characterization-Mössbauer Spectroscopy: The iron oxide phase was identified using room temperature ${ }^{57} \mathrm{Fe}$ Mössbauer spectroscopy. Approximately $50 \mathrm{mg}$ of each sample was mixed with ca. $200 \mathrm{mg}$ sucrose in a pestle and mortar to form a paste, and then flattened within a coin shaped absorber. Spectra were recorded at room temperature in transmission geometry using a ${ }^{57}$ Co source in a Rh matrix, using a W302 spectrometer and W202 detector, both from SEECo (Minneapolis, USA). Low temperature Mössbauer measurements were recorded also in transmission geometry with a source of ${ }^{57} \mathrm{Co}$ in $\mathrm{Rh}$, but using a close cycle helium refrigerator from APD Cryogenics and a spectrometer from Wissel $\mathrm{GmbH}$ operated in the constant acceleration mode. Best fits to the spectra have been obtained using a model independent analysis to obtain the best fit (lowest $\chi^{2}$ ) to the observed spectra as described by Fock et al. ${ }^{[57,69]}$ In this way, spectra have been fit using Voigtian lineshapes to represent a Gaussian distribution of Lorentzian hyper-fine fields, which we have used to account for a distribution of particle volumes and shapes as well as reduced hyperfine fields related to relaxation effects.

Characterization - Infrared Spectroscopy: FTIR was used to detect and identify the presence of coating on the nanoparticle surface using a Bruker IFS 66V-S in the range of $2000-250 \mathrm{~cm}^{-1}$. The samples were prepared by diluting the dried powder in $\mathrm{KBr}$ at $2 \%$ by weight and pressing it into a pellet.

Characterization-DLS Analysis: Colloidal properties were analysed by DLS. Measurements were carried out in an instrument NICOMP Submicron Particle Sizer Model 370. The measured angle was $90^{\circ}$, a wavelength of $632.8 \mathrm{~nm}$ was used, the temperature was set to $30{ }^{\circ} \mathrm{C}$ and the sample was diluted with 
MilliQ water to $5 \mathrm{mM}$ iron. Then, $300 \mu \mathrm{L}$ of the diluted sample were measured three times using a run time of $5 \mathrm{~min}$ each. The data analysis was performed with the 2 nd cumulant method, and the Gaussian distributions are intensity-weighted. For the Z-potential measurements, a Zetasizernano ZS, Malvern instrument was used and data were evaluated by the Smoluchowski model. The measurements were recorded at $25^{\circ} \mathrm{C}$. $700 \mu \mathrm{L}$ of each dilution (which was likewise diluted to $5 \mathrm{mM}$ iron with MilliQ water) was pipetted into a folded capillary cell (DTS 1062) and then measured two times with 20 runs per measurement.

Characterization-DC Magnetometry Measurements: DC magnetization measurements have been performed at $300 \mathrm{~K}$ on liquid samples using a Magnetic Property Measurement System (MPMS)-XL from Quantum Design (USA). $30 \mu \mathrm{L}$ of the suspensions were filled into a polycarbonate (PC) capsule. The measurement system was demagnetized before measurement and the magnetic moment was recorded within a magnetic field range of $\pm 4.9 \mathrm{~T}$. Data evaluation was performed by subtracting the empty sample holder signal (measured in a previous measurement) and the water contribution (using the susceptibility $\left.\chi_{\text {water }}=-9 \times 10^{-6}\right)$. The sample magnetization $\mathrm{M}^{\prime}$ was calculated using the measured magnetic moment and the suspension volume $M^{\prime}=$ Magn. moment $/ V$, and with the iron concentration $\mathrm{c}_{\mathrm{Fe}}$, the magnetization $M=M^{\prime} / c_{\mathrm{Fe}}$ in terms of $\mathrm{Am}^{2} / \mathrm{kg}(\mathrm{Fe})$ was obtained. Fitting the virgin curve by a bimodal model (see section 4 of SI) provides magnetic core size.

Characterization-AC Susceptibility Measurements: The altering current susceptibility (ACS) measurements on nanoflower samples were performed at room temperature utilizing two custom-built susceptometers by TU Braunschweig. ${ }^{[70]}$ The frequency was swept from $10 \mathrm{~Hz}-10 \mathrm{kHz}$ and from $200 \mathrm{~Hz}-1 \mathrm{MHz}$ in logarithmical steps. The amplitudes of the excitation field amounted to $567 \mu \mathrm{T}$ and $90 \mu \mathrm{T}$, respectively. Measurements have been carried out on suspensions of original and 10-fold diluted concentration and on freeze-dried reference samples, i.e. samples with immobilized nanoparticles of original concentration with a sample volume of $150 \mu \mathrm{L}$ each. A blank measurement was performed prior to the sample measurement and subtracted from the acquired spectra of the sample. The systems are calibrated with a $\mathrm{Dy}_{2} \mathrm{O}_{3}$ powder sample with defined volume susceptibility. We also used two AC susceptometers at Acreo, the first one is the commercially available DynoMag system with excitation frequencies up to $500 \mathrm{kHz}$ and a prototype high frequency $\mathrm{AC}$ susceptometer with excitation frequencies up to $10 \mathrm{MHz}$. In the DynoMag system the excitation field is 5 $\mathrm{mT}$ and $30 \mu \mathrm{T}$ in the high frequency AC susceptometer. Also in these AC susceptometers the calibration was performed using the paramagnetic $\mathrm{Dy}_{2} \mathrm{O}_{3}$ material.

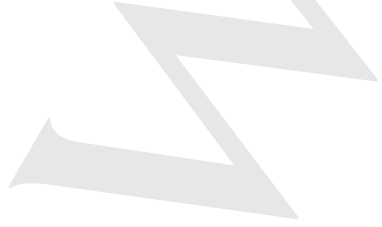

\section{Supporting Information}

Supporting Information is available from the Wiley Online Library or from the author.

\section{Acknowledgements}

This work was partially supported by the European Commission Framework Program 7 (NanoMag project, NO 604448) and by the Spanish Ministry of Economy and Competitiveness (Mago project, $\mathrm{N}^{\circ}$ MAT2014-52069-R).

\section{Conflict of Interest}

The authors declare no conflict of interest.

\section{Keywords}

colloids, magnetic properties, magnetite, nanoflowers, selfassembly

Received: March 9, 2017

Revised: April 4, 2017

Published Online: MM DD, YYYY

[1] P. Xing, Y. Zhao, Advanced Materials 2016, 28, 73049.

[2] S. Pan, L. He, J. Peng, F. Qiu, Z. Lin, Angewandte Chemie 2016, 30, 8459.

[3] M. Volkmann, M. Meyns, R. Lesyuk, H. Lehmann, C. Klinke, Chemistry of Materials 2016, 29, 726.

[4] V. Sayevich, B. Cai, A. Benad, D. Haubold, L. Sonntag, N. Gaponik, V. Lesnyak, A. Eychmüller, Angewandte Chemie International Edition 2016, 55, 6334.

[5] J. K. Stolarczyk, A. Deak, D. F. Brougham, Advanced Materials 2016 , 28, 5400 .

[6] Z. Nie, A. Petukhova, E. Kumacheva, Nature nanotechnology 2010, $5,15$.

[7] J. J. De Yoreo, P. U. Gilbert, N. A. Sommerdijk, R. L. Penn, S. Whitelam, D. Joester, H. Zhang, J. D. Rimer, A. Navrotsky, J. F. Banfield, Science 2015, 349, aaa6760.

[8] M. Ocaña, R. Rodríguez-Clemente, C. J. Serna, Advanced Materials 1995, 7, 212.

[9] M. Raju, A. C. Van Duin, K. A. Fichthorn, Nano letters 2014, 14, 1836.

[10] C. Frandsen, B. A. Legg, L. R. Comolli, H. Zhang, B. Gilbert, E. Johnson, J. F. Banfield, CrystEngComm 2014, 16, 1451.

[11] M. B. Bannwarth, S. W. Kazer, S. Ulrich, G. Glasser, D. Crespy, K. Landfester, Angewandte Chemie International Edition 2013, 52, 10107.

[12] B. Ingham, T. H. Lim, C. J. Dotzler, A. Henning, M. F. Toney, R. D. Tilley, Chemistry of Materials 2011, 23, 3312.

[13] S. Dutz, IEEE Transactions on Magnetics 2016, 52, 1.

[14] D. Serantes, K. Simeonidis, M. Angelakeris, O. Chubykalo-Fesenko, M. Marciello, M. P. Morales, D. Baldomir, C. Martinez-Boubeta, The Journal of Physical Chemistry C 2014, 118, 5927.

[15] U. Jeong, X. Teng, Y. Wang, H. Yang, Y. Xia, Advanced Materials 2007, $19,33$.

[16] L. Gutiérrez, R. Costo, C. Grüttner, F. Westphal, N. Gehrke, D. Heinke, A. Fornara, Q. Pankhurst, C. Johansson, S. VeintemillasVerdaguer, M. P. Morales, Dalton Transactions 2015, 44, 2943.

[17] M. A. Gijs, F. Lacharme, U. Lehmann, Chemical reviews 2009, 110 , 1518. 
[18] J. Nowak, F. Wiekhorst, L. Trahms, S. Odenbach, Journal of Physics: Condensed Matter 2014, 26, 176004.

[19] M. Mahmoudi, H. Hofmann, B. Rothen-Rutishauser, A. Petri-Fink, Chemical reviews 2011, 112, 2323.

[20] S. Laurent, D. Forge, M. Port, A. Roch, C. Robic, L. Vander Elst, R. N. Muller, Chemical Reviews 2008, 108, 2064.

[21] D. Eberbeck, C. L. Dennis, N. F. Huls, K. L. Krycka, C. Gruttner, F. Westphal, IEEE Transactions on Magnetics 2013, 49, 269.

[22] C. Grüttner, K. Müller, J. Teller, F. Westphal, International Journal of Hyperthermia 2013, 29, 777.

[23] S. Dutz, M. Kettering, I. Hilger, R. Müller, M. Zeisberger, Nanotechnology 2011, 22, 265102.

[24] L. Lartigue, P. Hugounenq, D. Alloyeau, S. P. Clarke, M. Lévy, J.-C. Bacri, R. Bazzi, D. F. Brougham, C. Wilhelm, F. Gazeau, ACS nano 2012, 6, 10935.

[25] C. Alexiou, W. Arnold, R. J. Klein, F. G. Parak, P. Hulin, C. Bergemann, W. Erhardt, S. Wagenpfeil, A. S. Luebbe, Cancer research 2000, 60, 6641

[26] N. C. Bigall, C. Wilhelm, M.-L. Beoutis, M. García-Hernandez, A. A. Khan, C. Giannini, A. Sánchez-Ferrer, R. Mezzenga, M. E. Materia, M. A. Garcia, Chemistry of Materials 2013, 25, 1055.

[27] G. Zoppellaro, A. Kolokithas-Ntoukas, K. Polakova, J. Tucek, R. Zboril, G. Loudos, E. Fragogeorgi, C. Diwoky, K. Tomankova, K. Avgoustakis, Chemistry of Materials 2014, 26, 2062.

[28] F. Mazuel, A. Espinosa, G. Radtke, M. Bugnet, S. Neveu, Y. Lalatonne, G. A. Botton, A. Abou-Hassan, C. Wilhelm, Advanced Functional Materials 2017, 27, 1605997.

[29] F. Ludwig, O. Kazakova, L. F. Barquín, A. Fornara, L. Trahms, U. Steinhoff, P. Svedlindh, E. Wetterskog, Q. A. Pankhurst, P. Southern, M. P. Morales, M. F. Hansen, C. Frandsen, E. Olsson, S. Gustafsson, N. Gehrke, K. Lüdtke-Buzug, C. Grüttner, C. Jonasson, C. Johansson, IEEE Transactions on Magnetics 2014, 50, 5300204.

[30] P. Mulvaney, W. J. Parak, F. Caruso, P. S. Weiss, ACS Publications 2016, 10, 9763.

[31] V. Schaller, G. Wahnström, A. Sanz-Velasco, P. Enoksson, C. Johansson, AIP Conference Proceedings 2010, 1311, 42.

[32] B. L. Frankamp, A. K. Boal, M. T. Tuominen, V. M. Rotello, Journal of the American Chemical Society 2005, 127, 9731.

[33] R. A. Bohara, N. D. Thorat, S. H. Pawar, RSC Advances 2016, 6, 43989.

[34] T. Sugimoto, E. Matijević, Journal of Colloid and Interface Science 1980, 74, 227.

[35] F. Vereda, J. de Vicente, M. P. Morales, F. Rull, R. Hidalgo-Álvarez, The Journal of Physical Chemistry C 2008, 112, 5843.

[36] G. A. Parks, Chemical Reviews 1965, 65, 177.

[37] M. A. Vergés, R. Costo, A. Roca, J. Marco, G. Goya, C. Serna, M. P. Morales, Journal of Physics D: Applied Physics 2008, 41, 134003.

[38] Y. Luengo, M. P. Morales, L. Gutiérrez, S. Veintemillas-Verdaguer, Journal of Materials Chemistry C 2016, 4, 9482.

[39] M. Bautista, O. Bomati-Miguel, X. Zhao, M. P. Morales, T. GonzalezCarreño, R. P. de Alejo, J. Ruiz-Cabello, S. Veintemillas-Verdaguer, Nanotechnology 2004, 15, S154.

[40] H. Dong, Y.-C. Chen, C. Feldmann, Green Chemistry 2015, 17, 4107.

[41] D. Caruntu, G. Caruntu, C. J. O'Connor, Journal of physics D: Applied physics 2007, 40, 5801.

[42] J. Ge, Y. Hu, M. Biasini, W. P. Beyermann, Y. Yin, Angewandte Chemie International Edition 2007, 46, 4342.

[43] P. Hugounenq, M. Levy, D. Alloyeau, L. Lartigue, E. Dubois, V. r. Cabuil, C. Ricolleau, S. p. Roux, C. Wilhelm, F. Gazeau, The Journal of Physical Chemistry C 2012, 116, 15702.

[44] W. Cai, J. Wan, Journal of colloid and interface science 2007, 305, 366.

[45] H.-L. Liu, S. P. Ko, J.-H. Wu, M.-H. Jung, J. H. Min, J. H. Lee, B. H. An, Y. K. Kim, Journal of Magnetism and Magnetic Materials 2007, 310,815 .
[46] J. Liu, Z. Sun, Y. Deng, Y. Zou, C. Li, X. Guo, L. Xiong, Y. Gao, F. Li, D. Zhao, Angewandte Chemie 2009, 121, 5989.

[47] Q. Sun, Z. Ren, R. Wang, W. Chen, C. Chen, Journal of Nanoparticle Research 2011, 13, 213.

[48] Y. Sahoo, A. Goodarzi, M. T. Swihart, T. Y. Ohulchanskyy, N. Kaur, E. P. Furlani, P. N. Prasad, The Journal of Physical Chemistry B 2005, 109, 3879.

[49] F. Vereda, M. P. Morales, B. Rodríguez-González, J. de Vicente, R. Hidalgo-Alvarez, CrystEngComm 2013, 15, 5236.

[50] C. Cheng, F. Xu, H. Gu, New Journal of Chemistry 2011, 35, 1072.

[51] J. Sommertune, A. Sugunan, A. Ahniyaz, R. S. Bejhed, A. Sarwe, C. Johansson, C. Balceris, F. Ludwig, O. Posth, A. Fornara, International journal of molecular sciences 2015, 16, 19752.

[52] V. Yathindranath, L. Rebbouh, D. F. Moore, D. W. Miller, J. van Lierop, T. Hegmann, Advanced Functional Materials 2011, 21, 1457.

[53] T. Yonezawa, K. Kamoshita, M. Tanaka, T. Kinoshita, Japanese Journal of Applied Physics 2008, 47, 1389.

[54] H. Okudera, K. Kihara, T. Matsumoto, Acta Crystallographica Section B: Structural Science 1996, 52, 450.

[55] C. Pecharromán, T. Gonzalez-Carreno, J. E. Iglesias, Physics and Chemistry of Minerals 1995, 22, 21.

[56] C. Frandsen, S. Mørup, Physical review letters 2005, 94, 027202.

[57] J. Fock, L. K. Bogart, O. Posth, M. F. Hansen, Q. A. Pankhurst, C. Frandsen, Hyperfine Interactions 2016, 237, 1.

[58] M. P. Morales, S. Veintemillas-Verdaguer, M. Montero, C. Serna, A. Roig, L. Casas, B. Martínez, F. Sandiumenge, Chemistry of Materials 1999, 11, 3058

[59] S.-H. Chung, A. Hoffmann, K. Guslienko, S. Bader, C. Liu, B. Kay, L. Makowski, L. Chen, Journal of applied physics 2005, 97, $10 \mathrm{R} 101$.

[60] S. Bogren, A. Fornara, F. Ludwig, M. P. Morales, U. Steinhoff, M. F. Hansen, O. Kazakova, C. Johansson, International journal of molecular sciences 2015, 16, 20308.

[61] F. Ludwig, T. Wawrzik, T. Yoshida, N. Gehrke, A. Briel, D. Eberbeck, M. Schilling, IEEE Transactions on Magnetics 2012, 48, 3780.

[62] M. Shliomis, V. Stepanov, Advances in Chemical Physics 1994, 87, 1.

[63] F. Ahrentorp, A. P. Astalan, C. Jonasson, J. Blomgren, O. T. Mefford, M. Yan, J. Cuortais, J. F. Berret, J. Fresnais, O. Sandre, S. Dutz, R. Müller, C. Johansson, in Aip Conference Proceedings 2010, 1311, 213.

[64] P. Svedlindh, T. Jonsson, J. García-Palacios, Journal of magnetism and magnetic materials 1997, 169, 323.

[65] D. Parker, I. Lisiecki, M. Pileni, The Journal of Physical Chemistry Letters 2010, 1, 1139.

[66] N. Gehrke, D. Heinke, D. Eberbeck, F. Ludwig, T. Wawrzik, C. Kuhlmann, A. Briel, IEEE Transactions on Magnetics 2015, 51, 1.

[67] M. Wabler, W. Zhu, M. Hedayati, A. Attaluri, H. Zhou, J. Mihalic, A. Geyh, T. L. DeWeese, R. Ivkov, D. Artemov, International Journal of Hyperthermia 2014, 30, 192.

[68] J. Rodríguez-Carvajal, Commission on powder diffraction (IUCr). Newsletter 2001, 26, 12.

[69] J. Fock, L. K. Bogart, D. Gonzalez-Alonzo, J. I. E. Martinez, M. F. Hansen, M. Varon, C. Frandsen, Q. A. Pankhurst, (In preparation).

[70] F. Ludwig, A. Guillaume, M. Schilling, N. Frickel, A. Schmidt, Journal of Applied Physics 2010, 108, 033918. 
Q1 APT to AU: Please provide postal code in affiliation 12.

Q2 APT to AU: Please provide the highest academic title (Dr./Prof.) for all authors, where applicable.

Q3 APT to AU: Please define all acronyms at their first appearance in the abstract, text and table of contents, respectively. Only expanded forms are allowed if the elements are cited only once in the article. 